\title{
La percepción de la inteligencia espiritual en las empresas
}

\section{The Perception of Spiritual Intelligence in Enterprises}

\section{JOSÉ BUSTELO GRACIA}

jbustelogracia@hotmail.com

Resumen: El presente artículo trata de establecer la situación actual en relación al conocimiento de las capacidades de la inteligencia espiritual y las practicas aplicadas para su desarrollo por parte de las personas capacitadas para implementar dichas prácticas en las organizaciones catalanas. El autor desarrolla los rasgos y características de la Inteligencia Espiritual que más pueden ser de utilidad en la empresa, es decir aquellas que pueden incidir en la implementación de un nuevo tipo de empresa más justa, más medioambiental y cuyos valores se acerquen más a la ética ciudadana.

Hay una fuerte presión de la sociedad actual para que las organizaciones asuman una serie de valores que van de lo social a lo ambiental y de lo ético a lo justo, los cuales están íntimamente relacionados con la Inteligencia Espiritual.

Palabras clave: Inteligencia Espiritual, Conocimiento espiritual, Espiritualidad laica, Empresa, Significado de la vida, Empatía.
Abstract: The present article aims to establish the current situation in relation to the knowledge of Spiritual Intelligence capacities and the practice needed to develop them by the people in charge to implement these practices on the Catalan organizations. The author develops the features and characteristics of Spiritual Intelligence that can be most useful in a corporation environment, those that can affect the implementation of a new type of business, fairer, more environmentally conscious and whose values are closer to citizen ethics.

There is strong pressure from today's society for organizations to assume a series of values that range from the social to the environmental and from the ethical to the fair, which are intimately related to Spiritual Intelligence.

Key words: Spiritual Intelligence, Spiritual Knowledge, Secular Spirituality, Business, Meaning of Life, Empathy. 


\section{INTRODUCCIÓN}

\section{Inteligencia Espiritual}

La inteligencia humana ha sido objeto de amplio debate por parte de la comunidad científica, la cual ha aportado varias formas de categorización y definiciones de este constructo. Mientras que la teoría clásica de la inteligencia $^{1}$ defiende la existencia de una inteligencia unitaria (para la cual Alfred Binet (1976) diseño una herramienta de medición, el cociente intelectual, que sigue siendo hoy en dia ampliamente utilizada), teorías más recientes, sin embargo, y con un creciente apoyo entre los autores científicos, hablan de la existencia de varias inteligencias ${ }^{2}$.

Howard Garner ${ }^{3}$ expuso la teoría de las inteligencias múltiples en la que hablaba de ciertas habilidades inherentes al ser humano cuyo desarrollo y práctica ampliaban y perfeccionaban los diferentes tipos de inteligencias. Entre estas Garner incluye las intrapersonales e interpersonales, cuyo desarrollo exige habilidad y práctica en la conciencia y emociones propias, la empatía y las "artes sociales", que es una forma de referirse a las relaciones con los demás. A pesar de que Garner siempre se resistió a incluir la inteligencia espiritual en su teoría, las intrapersonales e interpersonales se pueden consideran una aproximación a esta última.

Richard Wolman ${ }^{4}$ defendía "la capacidad humana para hacer preguntas sobre el significado de la vida" como una de las capacidades necesarias para el desarrollo de la inteligencia espiritual. Esta capacidad, para el psicólogo estadounidense, implica la respuesta a la necesidad humana de encontrar un objetivo trascendente que justifique la vida, que le dé significado y proporcione una ética personal y colectiva, es decir una ética ciudadana. La habilidad para transcender la realidad y hacerse preguntas existenciales de la vida que expresa Wolman fue también mencionada por Vaughan (2002). Otros autores, sin embargo, apuntan a la psicología de la personalidad al entender al ser humano

\footnotetext{
1 Riggio, R.E. (2002).

2 Gardner, H. E. (1983) o Sternberg, R. J. (1988).

3 Gardner definió la Inteligencia Espiritual como: "...la capacidad para situarse a sí mismo con respecto al cosmos, como la capacidad de situarse a sí mismo con respecto a los rasgos existenciales de la condición humana, como el significado de la vida, el significado de la muerte y el destino final del mundo físico y psicológico en profundas experiencias como el amor a otra persona o la inmersión en un trabajo de arte". Garner, H. (1993).

4 Wolman, R. (2003).
} 
como un conjunto holístico integrado por el temperamento que aúna los diferentes sistemas biológicos que estructuran el organismo ${ }^{5}$.

De modo unificador, Zohar y Marshall ${ }^{6}$, relacionan la Inteligencia Espiritual con la Emocional y Lógico-racional, de manera que las tres posibilitan afrontar la vida desde una gran capacidad de resiliencia y una comprensión holística para entender el significado y el sentido de los propios actos ${ }^{7}$. Para estos autores la Inteligencia Espiritual implica ser flexible, tener un alto índice de conciencia en sí mismo, afrontar y trascender el dolor y el sufrimiento, e inspirar y ser inspirado por valores éticos y colectivos.

Vaughan $^{8}$ también relaciona la Inteligencia Espiritual con la Emocional por cuanto lo espiritual y lo emocional están relacionados, y porque la espiritualidad significa el desarrollo de lo intrapersonal y lo interpersonal; para la psicóloga norteamericana la inteligencia espiritual implica que la persona es consciente de su relación con lo trascendente, es decir está íntimamente relacionada tanto con la tierra, o sea con el entorno, como con las personas que lo habitan. Para ella la inteligencia espiritual se revela cuando nos preguntamos ¿Quién soy?, ¿Qué hago yo aquí? ¿Qué es lo que realmente importa? Preguntas que trascienden del individuo y tratan de averiguar cuál es la esencia que nos rodea.

En cuanto a los esfuerzos científicos para aportar una herramienta útil a la hora de medir la Inteligencia Espiritual, Nasel (2004) propone una aplicación práctica de la Inteligencia Espiritual a los acontecimientos diarios de la vida y aporta una Escala de 17 Items para medir la Inteligencia Espiritual. Sin embargo, su modelo no puede ser implementado de forma global al estar basado en gran medida en valores cristianos que excluyen otros valores universales. Amram (2009) aporta una escala de 22 items agrupados en cinco dimensiones: Conciencia, significado, transcendencia, verdad y gracia, sin embargo, su modelo no hace una distingcion clara entre las dimensiones propuestas de: Valor, Producción de Significado Personal, Conciencia Transcendental y Expansión del estado consciente.

\footnotetext{
5 Cloninger, C.R. (2004).

6 Zohar, D. y Marshall, I. (1997).

7 Zohar y Marshall realizaron experimentos para averiguar la impresión que ejercía la Inteligencia Espiritual en el cerebro humano a través del análisis de las ondas electromagnéticas. Encontraron que cuando la persona hablaba sobre el sentido de la vida o las prácticas espirituales se producían respuestas de hasta cuarenta megahercios en las neuronas.

8 Vaughan, R. (2003).
} 
En la misma línea que Nasel, Torralba ${ }^{9}$ habla de una inteligencia espiritual que está por encima de las religiones o creencias concretas, superando estas para convertirse en una dimensión espiritual que determina el objetivo último del ser como individuo o como colectivo. El filósofo catalán plantea que la Inteligencia Espiritual "permite acceder a los significados profundos, plantearse los fines de la existencia y las más altas motivaciones de ésta. Es la inteligencia del yo profundo la que se enfrenta a las graves cuestiones de la existencia y, a través de ella, busca respuestas creíbles y razonables" ${ }^{10}$. Por otra parte, también entiende que la materia es un componente de la realidad, pero no el único. No todo es materia, sino que existe la energía que complementa a dicha materia: "el materialismo es la cárcel del pensamiento, su negación" tiene como máxima, y añade: "no hay filosofía si no hay espiritualidad; no hay cultura sin espiritualidad"11.

Así pues, la Inteligencia Espiritual se enmarca dentro de la teoría de las inteligencias múltiples y se identifica por requerir la presencia de al menos 3 dimensiones:

a. Dimensión cognitiva que desarrolla el conocimiento espiritual.

Esta dimensión incluye el Razonamiento moral, que es la capacidad de la persona de interiorizar conceptos éticos, personales y sociales, a partir de un aprendizaje sobre los principios universales de la igualdad, justicia, no discriminación y los derechos comunes a toda la humanidad. Estos principios se adquieren por medio del razonamiento y el análisis que permite al individuo organizar su vida alrededor de un sistema de valores presidido por la Inteligencia Espiritual. La Meditación o reflexión periódica sobre los valores universales y las relaciones con los semejantes, llegando a la mayor profundidad posible de la conciencia de cada uno de los individuos. La Búsqueda del sentido que implica preguntarse sobre la existencia, su principio, desarrollo, objetivo de la misma y relacionar los actos cotidianos con el principio y el fin. Es decir, dedicar un tiempo al análisis de la trascendencia del individuo. El Autoconocimiento o ser consciente de la realidad del entorno y las características propias que inciden y se retroalimentan de dicho entorno. La Autotrascendencia que es la capacidad de buscar más allá de sí mismo y la Resiliencia o capacidad de

\footnotetext{
9 “...a pesar del progreso que se ha hecho en los últimos años, existen todavía reticencias y dificultades para reconocer la dimensión espiritual de la persona. El materialismo teórico y práctico es el más grande obstáculo para reconocerlas, pues reduce el ser humano a puro cuerpo”. Torralba, F. (2013).

10 Torralba, F. (2013).

11 Torralba, F. (2013).
} 
enfrentarse al sufrimiento y sobreponerse a las desgracias de todo tipo que puedan afectar al individuo. El dolor es parte de la vida y, por tanto, una oportunidad de aprendizaje y superación.

b. Dimensión afectiva.

Esta dimensión incluye el Entusiasmo en realizar en cada momento aquella actividad en que uno esté incurso de manera motivada y plenamente. La Estética que implica gozar de las formas artísticas en toda su extensión. Es decir, ser capaz de distinguir lo bello, aprehenderlo y entusiasmarse en su contemplación. La Comprensión por el misterio como una forma de investigación de la vida, buscar en lo irracional la emoción y el placer en comprender lo intrigante. La Empatía, que trata de la capacidad de integrarse en las emociones de otros. La Tranquilidad para encontrar la paz interior, lo cual depende del conocimiento de sí mismo, confianza en las capacidades propias, esperanza en poder alcanzar los objetivos y estabilidad espiritual. La Felicidad, que consiste en sentirse de acuerdo con uno y con el entorno, con todos, es un momento, una sensación de plenitud.

c. Dimensión conductual.

Esta dimensión incluye el Control de los impulsos; es la capacidad de respuestas de acuerdo con el propio sentido que cada uno le da a la vida. Implica, en todo momento, proporcionar respuestas a uno mismo y al exterior de acuerdo con la personalidad de uno. El Estilo de vida define cómo se plantea la vida en función de los agentes externos que inciden en cada persona: consumo, modas, tendencias y todo ello contrastado con los valores personales. La Salud se refiere a las conductas que puedan poner en peligro la salud o aquellas, por lo contrario, que preserven esta y se protejan frente a actividades o costumbres que la socaven. El cuidado del cuerpo siempre ha sido considerado un paso importante para el desarrollo espiritual. La Solidaridad es una mezcla de autotrascendencia y empatía dirigida a otro u otros. $\mathrm{Y}$ un valor muy necesario en cualquier sociedad ${ }^{12}$.

\section{Inteligencia Espiritual en la Sociedad}

La Inteligencia Espiritual es una capacidad humana y, por tanto, intrínseca del ser humano tal y como defienden varios autores ${ }^{13}$, sin embargo, esta

12 Emmmons, R. A. (2000), pp. 57-64 y Arias, R. y Lemos, V. (2015), núm. 1.

13 Moberg, D. O. (2002); Wigglesworth, C. (2013). 
capacidad, al igual que todas las demás, necesita de una inversión y entrenamiento constantes para alcanzar su máximo potencial. Sin embargo, la gran mayoría de la educación actual, desde la misma Primaria, tiende a promocionar la inteligencia formal-matemática y sólo en algunos casos, se ha abierto cierto margen a la inteligencia emocional. Solo en los últimos años se ha comenzado a plantear la posibilidad de educar desde la perspectiva de la Inteligencia Espiritual. El filósofo francés Jacques Maritain ${ }^{14}$ apuntó a la necesidad de introducir la espiritualidad en la educación ya que la espiritualidad era la base de la educación y el objetivo de la educación, es la formación de la persona. Dentro de este planteamiento la educación espiritual se revela como una dimensión holística de la educación, es decir que prepara para cualquier desarrollo personal en la sociedad y en cualquiera de sus campos.

Por lo anteriormente estipulado, la falta de inversión en el desarrollo de la Inteligencia espiritual se transmite al resto de los ámbitos humanos. La exclusión de la espiritualidad en las actividades sociales ha sido discutida por varios autores ${ }^{15}$ que hacen referencia a la necesidad de recuperar la relación entre el medio ambiente y el ser humano. Esto se fundamenta en que la espiritualidad es entendida, por estos autores, como un rasgo propio del ser bumano, que impregna toda relación entre la persona y el entorno, y las otras personas que componen dicho entorno, es decir afecta a todas las relaciones sociales del individuo.

La Espiritualidad individual es expresada por Torralba ${ }^{16}$ como aquella que trata de profundizar en las relaciones con otras personas y prioriza lo que les une a ellas sobre lo que les separa, relativizando las diferencias, es decir la espiritualidad propia lleva a las personas a transformar las experiencias con otras personas y moldean sus comportamientos. En este sentido algunos autores han profundizado en la manera en la que las prácticas organizativas pueden modelar la espiritualidad de los trabajadores/estudiantes ${ }^{17}$. Sin embargo, a nivel de las organizaciones la espiritualidad debe implementarse como parte de la misión, valores y políticas de la organización cuyo desarrollo en el tejido empresarial promueva practicas espirituales. McGhee y Grant 2017 apuntan que cualquier cultura espiritual emerge de los individuos que conforman dicha cultura.

\footnotetext{
14 Maritain, J. (1998).

15 Puig Baguer, J.; Echarri Iribarrren, F. y Casas Jericó, M. (2014), pp. 115-140.

16 Torralba, F. (2010) núm. 59.

17 Pfeffer, J. (2003).
} 


\section{La Inteligencia Espiritual en la empresa.}

La II Guerra Mundial marcó un antes y un después en prácticamente todas las actividades que de una manera u otra caracterizaban a la sociedad occidental. En el ámbito organizacional se implementaron las Nuevas Tecnologías (NN.TT.) que revolucionaron la forma y estructura del trabajo. Junto con los nuevos objetivos y técnicas también se inició un cambio en las relaciones internas y externas de la empresa, el auge de la capacidad del consumidor como elemento de presión en las empresas, y ante la amenaza de no consumir el producto creado, obligó a estas a cambiar su conducta. A medida que la cultura empresarial modificaba sus criterios y objetivos para adaptarse a los cambios de la sociedad, la espiritualidad se fue haciendo un hueco en la cultura organizativa. Karakas (2010), como parte de su extensiva revisión literaria apunta que en aquellas empresas donde la espiritualidad ésta implementada dentro de los departamentos de recursos humanos, la calidad laboral y el bienestar de los trabajadores es mayor que en otras empresas. Este autor también muestra que en aquellas empresas en donde la espiritualidad está presente, de una forma u otra, la sensación de comunidad y de pertenencia aumenta entre los trabajadores.

Wang, D. y Hsieh, C. (2013) ya mencionaron las consecuencias negativas creadas por una cultura de trabajo equivocada. En la empresa la definición e implementación de una cultura de trabajo sólida y sana es esencial para el desarrollo y crecimiento de la misma. La pregunta que se hace el investigador es si la Inteligencia Espiritual tiene una aplicación práctica; si la enseñanza que se ha desarrollado sobre la base de un componente ético como la Inteligencia Espiritual, puede ser el fundamento del desarrollo de toda una praxis que permita su aplicación a las realizaciones humanas, por ejemplo, la empresa.

Mientras que el valor de la aplicación de la inteligencia emocional en la empresa ha sido ampliamente estudiado en la literatura ${ }^{18}$, no existen muchos estudios que exploren la relación entre la inteligencia espiritual y el mundo laboral. Entre los estudios llevados a cabo en este campo destacan los de Punia y Yadav (2013) y (2015) los cuales relacionan la inteligencia emocional con la inteligencia espiritual y proponen nuevas líneas de investigación basadas en la relación positiva de la inteligencia emocional con el Comportamiento Ciudadano de la Organización para aplicar la inteligencia espiritual en la empresa.

18 Wright, P.; Gardner, T. y Moynihan, L. (2003); Lee, F.; Lee, T. y Wu, W. (2010); Kim, A. y Lee, C. (2012). 
Si bien es cierto que la Inteligencia emocional incide, según Ferreira ${ }^{19}$, en el tipo de liderazgo, motivación, compromiso, actitudes, cultura organizacional, calidad de comunicación y resolución de conflictos en el marco de una organización, uno de los problemas fundamentales es que en pleno siglo XXI todavía es necesario preguntarse si la sociedad empresarial actual conoce qué es la Inteligencia Espiritual asociada al sector de la empresa; si la empresa percibe la Inteligencia espiritual como una ayuda en las estrategias y tácticas empresariales, en las relaciones humanas, en los tratos con proveedores y clientes, en el manejo de las finanzas y, de manera especial, en la economía justa y colaborativa.

Cierto que una empresa está organizada en torno a rutinas y procedimientos repetitivos en los que, de estar bien organizada, cada uno de sus miembros tiene una función definida y sabe, o debe saber, desarrollar su cometido en cada instante y en función de las necesidades empresariales, por lo que la función de la Inteligencia Espiritual no está en la atribución de responsabilidades ni en la ejecución de las mismas, es decir en los engranajes de la corporación, sino en los planteamientos, interrelaciones y comportamientos, es decir los fluidos que permiten el funcionamiento de dichos engranajes.

Con todo lo anterior en mente este artículo explora el estado de la implementación de la Inteligencia Espiritual en las organizaciones catalanas a través de los conocimientos y actitudes de los profesionales de estas organizaciones en cuanto a la Inteligencia Espiritual y a las actividades llevadas a cabo para su promoción dentro de sus respectivas organizaciones.

19 Ferreira, E. (2014), pp. 194-200. 


\section{DISEÑO DEL ESTUDIO}

\section{Método}

El presente artículo incluye un estudio de carácter transversal, observacional, descriptivo y analítico. La herramienta de análisis es un cuestionario que fue enviado a profesionales de organizaciones catalanas con una posición en las mismas lo suficientemente elevada como para influir en la cultura, objetivos y sistema de relaciones laborales. El perfil de los profesionales a los que se les envió el cuestionario incluye: Directores de Colegios, Decanos de universidad, CEO, CFO, Directores de Recursos Humanos, etc. El nivel más alto de responsabilidad organizacional fue buscado en todo momento. La muestra final incluye a 365 directivos de diversas organizaciones catalanas.

El cuestionario enviado fue diseñado para analizar el grado de implementación de la Inteligencia Espiritual en las organizaciones en las que trabajan los participantes en la muestra. Para ello el cuestionario se diseñó con un apartado que incluye una sección de datos demográficos y las siguientes preguntas:

¿Considera que el desarrollo de la inteligencia espiritual en su organización mejoraría las capacidades de sus empleados?

¿Desarrolla alguna actividad en su organización para la promoción de la inteligencia espiritual?

Las organizaciones se han dividido entre Empresas, Escuelas de Negocio, Escuelas/Colegios y Universidades.

\section{Resultados}

Los datos demográficos con respecto al tipo de organización al que pertenecen los participantes de la muestra se resumen en la tabla 1. Los participantes que en mayor número respondieron al cuestionario forman parte del mundo empresarial (65,8\%), seguidos de las Escuelas/Colegios (15,4\%), las Universidades (12,9\%) y las Escuelas de Negocio (5,8\%). 
Tabla 1. Tipo de Organización.

\begin{tabular}{lcc} 
& Frecuencia & Porcentaje \\
\hline Empresa & 239 & 65,8 \\
\hline Escuela de Negocios & 21 & 5,8 \\
\hline Escuela/Colegio & 56 & 15,4 \\
\hline Universidad & 47 & 12,9 \\
\hline Total & 363 & 100,0 \\
\hline
\end{tabular}

Como vemos en la tabla 2 el desconocimiento de las capacidades del desarrollo de la inteligencia espiritual es amplio entre la muestra; un 46,3\% de la misma desconoce estas capacidades, mientras que sólo un 4,7\% de la muestra las conoce, pero las encuentra irrelevantes en el marco laboral. Un dato significativo es que más de la mitad de la muestra, un 53,7\%, dice conocer las capacidades de la Inteligencia Espiritual, lo que supone un amplio porcentaje.

Tabla 2. ¿Considera que el desarrollo de la inteligencia espiritual en su organización mejoraría las capacidades de sus estudiantes/empleados?

Frecuencia Porcentaje

\begin{tabular}{lrr}
\hline $\begin{array}{l}\text { Desconozco las capacidades del desarrollo de la } \\
\text { inteligencia espiritual. }\end{array}$ & 168 & 46,3 \\
\hline Sí, las considero importantes e intento desarrollarlas. & 178 & 49,0 \\
\hline No las considero importantes dentro de mi organización. & 17 & 4,7 \\
\hline Total & 363 & 100,0 \\
\hline
\end{tabular}

En cuanto a si el participante desarrolla alguna actividad para la promoción de la inteligencia espiritual, el 46,8\% de los encuestados respondió "No dispongo de información o conocimiento para hacerlo", lo que implica que, si estuvieran en posesión de estas herramientas, las introducirían en su organización. 
Tabla 3. ¿Desarrolla alguna actividad en su organización para la promoción de la inteligencia espiritual?

Frecuencia Porcentaje

\begin{tabular}{lrc}
\hline No dispongo de información o conocimiento para hacerlo & 170 & 46,8 \\
\hline Sí & 83 & 22,9 \\
\hline No & 108 & 29,8 \\
\hline N/A & 2 &, 6 \\
\hline Total & 363 & 100,0
\end{tabular}

Con el objetivo de explorar la relación ente el tipo de organización y la variable que responde a la pregunta ¿Considera que el desarrollo de la inteligencia espiritual en su organización mejoraría las capacidades de sus empleados? se llevó a cabo un análisis con el Test estadístico Chi al cuadrado. El resultado del test muestra que no existe una diferencia estadísticamente significante $(6)=10,145 ; p=0,119$. Así pues, se concluye que las variantes son independientes entre sí.

Los participantes cuyo lugar de trabajo es la Empresa son los que muestran un mayor desconocimiento de las capacidades de desarrollo de la Inteligencia Emocional ( $\mathrm{N}=118)$. Esto supone un 70,2\% de todas las contestaciones negativas en esta esta variable. Sin embargo, es alentador ver que en 111 casos los participantes dentro del grupo de empresas contestaron que sí consideraban estas capacidades importantes, lo que supone un $62,4 \%$ de todas las contestaciones positivas de esta variable. En el gráfico 1 de barras se muestra la relación gráfica de los datos. 


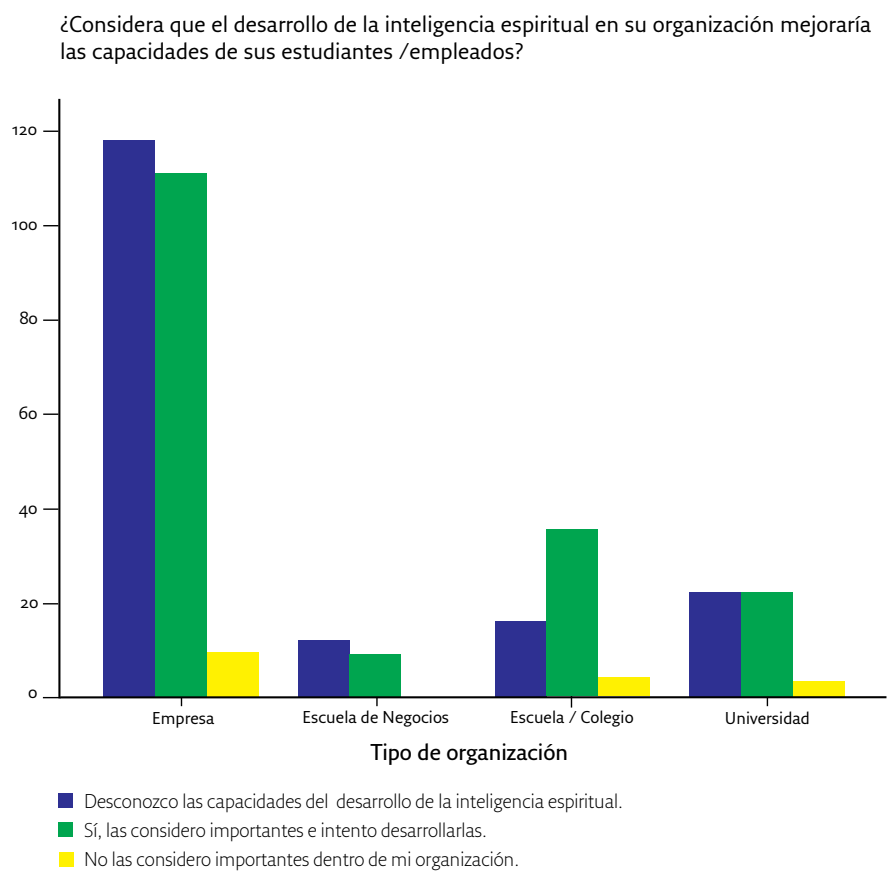

Se ha realizado el mismo análisis estadístico para analizar la relación entre las variables ¿Considera que el desarrollo de la inteligencia espiritual en su organización mejoraría las capacidades de sus empleados? Y ¿Desarrolla alguna actividad en su organización para la promoción de la inteligencia espiritual? El resultado del test muestra que existe una diferencia estadísticamente significante entre las variantes $(4)=122,557 ; \mathrm{p}=0,000$.

En la tabla 4 se muestran las relaciones entre las variables. Así pues, vemos que 118 participantes que contestaron desconocer las capacidades de la inteligencia espiritual también contestaron de manera coherente no disponer de información para desarrollar alguna actividad en su organización para la promoción de la inteligencia espiritual; sólo 3 participantes contestaron que desarrollar alguna actividad, lo que implica una falta de atención y/o comprensión a las preguntas del cuestionario; y 47 participantes que contestaron que no conocen las capacidades para el desarrollo de la inteligencia espiritual 
respondieron, también en este caso de manera coherente, que no desarrollan ninguna actividad para su promoción. Entre aquellos que consideraban importante el desarrollo de la inteligencia espiritual, 51 casos no disponen de la información necesaria para desarrollar alguna actividad en su organización para la promoción de la inteligencia espiritual, 78 participantes desarrollan algún tipo de actividad y 47 consideran importantes las capacidades del desarrollo de la inteligencia espiritual, pero no desarrollan ninguna actividad destinada a su promoción. Entre aquellos participantes que no consideran importante el desarrollo de la inteligencia espiritual solo 1 caso contestó que no disponían de información necesaria para desarrollar alguna actividad en su organización para la promoción de la inteligencia espiritual; en dos casos los participantes no encuentran estas capacidades importantes, pero sí que aplican algún tipo de actividad para su desarrollo lo que indica, otra vez, una falta de atención y/o comprensión a las preguntas del cuestionario.

Tabla 4. Relaciones entre las variables.

\begin{tabular}{l|c|c|c|c} 
& $\begin{array}{l}\text { Desconozco las } \\
\text { capacidades del } \\
\text { desarrollo de la } \\
\text { inteligencia } \\
\text { espiritual. }\end{array}$ & $\begin{array}{l}\text { Sí, las considero } \\
\text { importantes e } \\
\text { intento desarro- } \\
\text { llarlas. }\end{array}$ & $\begin{array}{l}\text { No, las considero } \\
\text { importantes } \\
\text { dentro de mi } \\
\text { organización. }\end{array}$ & Total \\
\hline $\begin{array}{l}\text { No dispongo } \\
\text { de información o } \\
\text { conocimiento } \\
\text { para hacerlo }\end{array}$ & 118 & 51 & 1 & 170 \\
\hline $\begin{array}{l}\text { Sí } \\
\text { No }\end{array}$ & 3 & 78 & 2 & 83 \\
\hline
\end{tabular}

Las limitaciones de este estudio se concentran en gran parte en la restricción del tipo de organización y el área elegida para su implementación, Cataluña. Los autores proponen estudios futuros que amplíen el marco de actuación del estudio, tanto geográfico como del tipo de participante en el estudio. 


\section{CONCLUSIÓN}

La inteligencia espiritual para Zohar y Marshall ${ }^{20}$ se refiere al sentido global de la vida de las personas, pero también a las relaciones entre lo personal y lo social, entre lo endógeno y lo exógeno, entre lo creativo y lo intelectivo, entre el soma y la psique. La globalización de la empresa actual en un mundo en el que se han reducido las distancias gracias a las NN.TT. y se han abaratado los costes, ha producido un cambio estructural en la sociedad y, consecuentemente, todas sus organizaciones. Entre ellas, en primer lugar, por su importancia, la empresa. Esta reestructuración de las corporaciones se sustenta en varios factores entre los cuales sería beneficioso incluir la Inteligencia Espiritual ${ }^{21}$. El presente estudio, sin embargo, pone de manifiesto que la Inteligencia espiritual sigue siendo una gran desconocida entre los directivos de las organizaciones y que en gran medida estas no implementan actividades que favorezcan su desarrollo. Sin embargo, varios estudios apuntan a un incremento de prácticas espirituales en la empresa ${ }^{22}$ lo que muestra pasos en la dirección correcta.

\section{BIBLIOGRAFÍA}

Amran, Joseph Yosi (2009), The Contribution of Emotional and Spiritual Inteligence to Effective Business Leadership, Institute of Transpersonal Psychology Working Paper, Palo Alto, California. Phd Dissertation.

Arias, Rodrigo y Lemos, Viviana (2015), “Una aproximación teórica y empírica al constructo de inteligencia espiritual”, Enfoques, vol. 27, n ${ }^{\circ}$ 1, pp. 79-102.

Binet, Alfred (1976), The Mind and the Brain, F. Legges, París.

Caduto, Michael J. (1998), "Ecological Education A System Rooted in Diversity”, The Fournal of Environmental Education, vol. 29, nº 4, pp. 11-16.

Cloninger, C. Robert (2004), Feeling Good: The Science of well-being, Oxford University Press, Nueva York.

20 Zohar, D. y Marshall, I (1997).

21 Mitroff, I. I. y Denton, E. A. (1999).

22 Gul, G. A. y Doh, J. (2004); Pfeffer, J. (2003). 
Cohen, Jacob (1988), Statistical Power and Analysis for the Behavioral Sciences, Lawrence Erlbaum Associates, Hillsdale.

Emmons, Robert A. (2000), "Spirituality and Intelligence: Problems and Prospects", The International fournal for the Psychology of Religion, vol. 10, $\mathrm{n}^{\circ} 1$, pp. 57-64.

Ferreira. Emilio (2014), "Inteligencia espiritual en la organización del siglo XXI", Revista Científica Ciencias Humanas, vol. 10, n 29, pp. 194-200.

Friedman, Thomas L. (2008), Hot, Flat, and Crowded: Why We Need a Green Revolution and How It Can Renew America, Farrar, Straus and Giroux, Nueva York.

Gallegos, Ramón (2005), Inteligencia Espiritual, Fundación Internacional para la Educación Holística, Guadalajara.

Gardner, Howard E. (1993), Multiple Intelligences. The Theory in Practice, Basic Books, Nueva York.

Grossman, Robert J. (2008), "Religion at Work", HR Magazine, Alexandria, vol. 53, nº 12 , pp. 26-34.

Gul, Gregory A. y Doh, Jonathan (2004), "The Transmutation of the Organization: Toward a More Spiritual Workplace”, fournal of Management Inquiry, vol. 13, n⿳0 12, pp. 128-139.

Hedlund-de Witt, Annick (2013), "Pathways to Environmental Responsability: A Qualitative Exploration of the Spiritual Dimension of Nature Experience", Fournal for the Study of Religion, Nature and Culture, vol. 72, $\mathrm{n}^{\circ} 2$, pp. 154-186.

Karakas, Fahri (2010), "Spirituality and Performance in Organizations: A Literature Review", Journal of Business Ethics, vol. 94, n 1, pp. 89-106. DOI:10.1007/s10551-009-0251-5.

Kim, Andrea y Lee, Choonwoo (2012), "How does HRM Enhance Strategic Capabilities? Evidence from the Korean Management Consulting Industry", The International Fournal of Human Resource Management, vol. 23, $\mathrm{n}^{\circ} 1$, pp. 126-146. DOI: https://doi.org/10.1080/09585192.2011.561247.

Lee, Feng-Hui; Lee,Tzai-Zang y Wu, Wann-Yih (2010), “The Relationship Between Human Resource Management Practices, Business Strategy and Firm Performance: Evidence from Steel Industry in Taiwan”, The Inter- 
national fournal of Human Resource Management, vol. 21, $\mathrm{n}^{\circ}$ 9, pp. 1351-1372. DOI: https://doi.org/10.1080/09585192.2010.488428.

Maritain, Jacques (1988), "Pour une philosophie de l'education", en Oeuvres completes de facques et Raissa Maritain (1939-1943), vol. VII. Editions Universitaires, Fribourg.

Marques, Joan (2008), "Spirituality at Work: Internal Growth with Potential External Challenges", The fournal for Quality and Participation, vol. 31, $\mathrm{n}^{\mathrm{o}} 3$, pp. 24-27.

McGhee, Peter y Grant, Patricia (2017), "Using Spiritual Intelligence to Transform Organisational Cultures", Electronic Fournal of Business Ethics and Organization Studies, vol. 22, n 1, pp. 4-14.

Mitroff, Ian I. (2003), "Do not Promote Religion under the Guise of Spirituality", Organization, vol. 10, n 2, pp. 375-382.

Mitroff, Ian I. y Denton, Elizabeth A. (1999), A Spiritual Audit of Corporate America: a Hard Look at Spirituality, Religion and Values in the Workplace, Jossey Pass, San Francisco.

Moberg, David O. (2002), "Assessing and Measuring Spirituality: Confronting Dilemmas of Universal and Particular Evaluative Criteria", Fournal of Adult Development, vol. 9, nº 1, pp. 47-60. DOI:10.1023/A:1013877201375.

Nasel, Dagmar Dasha (2004), Spiritual Orientation in Relation to Spiritual Intelligence: A New Consideration of Christianity and New Age Individualistic Spirituality, University of South Australia, Adelaida, Doctoral Dissertation. Available from:https://www.researchgate.net/publication/321875385_Spiritual_intelligence_An_overview.

Pfeffer, Jeffrey (2003), "Business \& Spirit: Management Practices that Sustain Values", en Giacalone, Robert A. \& Jurkiewicz, Carole L. (Eds.), The Handbook of Workplace Spirituality and Organizational Performance, M.E. Sharpe, Armonk, pp. 27-43.

Puche, José Daniel (2002), Desarrolle su inteligencia espiritual con la PNL, Intermedio, Bogotá.

Puig Baguer Jordi; Echarri Iribarren, Fernando y Casas Jericó, María (2014), "Educación ambiental, Inteligencia Espiritual y Naturaleza", Teoría de la Educación, vol. 26, nº 2, pp. 115-140. 
Punia, Bijender Kumar y Garg, Naval (2014), "An Organisational Analysis of High Performance Work Practices", Asian fournal of Management, vol. $5, \mathrm{n}^{\circ} 3$, pp. 318-324.

Punia, Bijender Kumar y Yadav, Priyanka (2015), "Predictive Estimates of Employees' Intelligence at Workplace with Special Reference to Emotional and Spiritual Intelligence, BIFIT-BVICAM's International Fournal of Information Technology, vol. 7, $\mathrm{n}^{\circ}$ 1, pp. 845-852.

Riggio, Ronald E. (2002), "Multiple Intelligences and Leadership: an Overview”, en Riggio, Ronald E.; Murphy, Susan E. y Pirozzolo, Francis J. (Eds.), Multiple Intelligences and Leadership, Erlbaum, Mahwah, NJ, pp. 1-6.

Sternberg, Robert J. (1988), The Triarchic Mind: A New Theory of Human Intelligence, Penguin Viking, New York.

Teijero, Sergio (2016), Inteligencia Espiritual. La suprema de las Inteligencias, Universidad Central, Caracas.

Torralba, Françesc (2010), "Cultivar la Inteligencia Espiritual”, Revista Mente Sana, no 59 , pp. 2-5. celona.

Torralba, Françesc (2013), Inteligencia espiritual, Plataforma Actual, Bar-

Vaughan, Frances (2002), "What is Spiritual Intelligence?”, fournal of Humanistic Psychology, vol. 42, n 2, pp. 16-33.

Wang, Dan-Sang y Hsieh, Chia-Chun (2013), "The Effect of Authentic Leadership on Employee Trust and Employee Engagement", Social Behavior or Personality: An International fournal, vol. 41, $\mathrm{n}^{\mathrm{o}}$ 4, pp. 613-624. https://doi.org/10.2224/sbp.2013.41.4.613.

Wigglesworth, Cindy (2013), "Spiritual Intelligence", en Neal, Judy (Ed.), Handbook of Faith \& Spirituality in the Workplace. Emerging Research \& Practice, Springer, Nueva York. DOI:10.1007/978-1-4614-5233-1_27.

Wolman, Richard (2003), Pensar con el alma, Obelisco, Barcelona.

Wright, Patrick; Gardner, Timothy y Moynihan, Lisa (2003), The Impact of HR Practices on the Performance of the Business", Human Resource Management Fournal, vol. 13, n ${ }^{\circ} 3$, pp. 21-36. DOI: https://doi.org/10.1111/ j.1748-8583.2003.tb00096.x.

Zohar, Danah y Marshall, Ian (1997), Inteligencia espiritual. La inteligencia que permite ser creativo, tener valores y fe, Plaza y Janés, Barcelona. 
